\title{
High-dose thiamine therapy counters dyslipidaemia in streptozotocin-induced diabetic rats
}

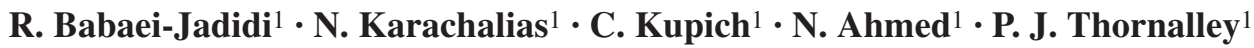 \\ ${ }^{1}$ Department of Biological Sciences, University of Essex, Colchester, Essex, UK
}

\begin{abstract}
Aims/hypothesis. Cardiovascular disease in diabetes is linked to increased risk of atherosclerosis, increased levels of triglyceride-rich lipoproteins and enhanced hepatic lipogenesis. The hepatic hexosamine pathway has been implicated in signalling for de novo lipogenesis by the liver. In this study, we assessed if decrease of flux through the hexosamine pathway induced by high-dose thiamine therapy counters diabetic dyslipidaemia.

Methods. The model of diabetes used was the streptozotocin-induced diabetic rat with maintenance insulin therapy. Normal control and diabetic rats were studied for 24 weeks with and without oral high-dose therapy ( 7 and $70 \mathrm{mg} / \mathrm{kg}$ ) with thiamine and benfotiamine. Plasma total cholesterol, HDL cholesterol and triglycerides were determined at 6-week intervals and hepatic metabolites and transketolase activity after death of the rats at 24 weeks.
\end{abstract}

Results. We found that thiamine therapy $(70 \mathrm{mg} / \mathrm{kg}$ ) prevented diabetes-induced increases in plasma cholesterol and triglycerides in diabetic rats but did not reverse the diabetes-induced decrease of HDL. This was achieved by prevention of thiamine depletion and decreased transketolase activity in the liver of diabetic rats. There was a concomitant decrease in hepatic UDP-N-acetylglucosamine and fatty acid synthase activity. Thiamine also normalised food intake of diabetic rats. A lower dose of thiamine $(7 \mathrm{mg} / \mathrm{kg})$ and the thiamine monophosphate prodrug benfotiamine (7 and $70 \mathrm{mg} / \mathrm{kg}$ ) were ineffective.

Conclusions/interpretation. High-dose thiamine therapy prevented diabetic dyslipidaemia in experimental diabetes probably by suppression of food intake and hexosamine pathway signalling but other factors may also be involved. Benfotiamine was ineffective.

Keywords Cholesterol · Dyslipidaemia ·

Glycation · Hexosamine - Streptozotocin · Thiamine · Triglycerides
Received: 8 April 2004 / Accepted: 13 July 2004

Published online: 11 December 2004

(C) Springer-Verlag 2004

P. J. Thornalley (

Department of Biological Sciences, University of Essex,

Wivenhoe Park, Colchester, Essex, CO4 3SQ, UK

E-mail: thorp@essex.ac.uk

Tel.: +44-1206-873010, Fax: +44-1206-873010

Abbreviations: 1,3-bis-PG, 1,3-bisphosphoglycerate $\cdot$ ACC, acetyl-CoA carboxylase $\cdot \mathrm{CEL}, N_{\varepsilon}$-(1-carboxyethyl)lysine $\cdot$ CML, $N_{\varepsilon}$-carboxymethyl-lysine $\cdot$ CTEP, cholesteryl ester transfer protein - DAG, diacylglycerol $\cdot$ DHAP, dihydroxyacetonephosphate $\cdot$ F-1,6-bis-P, fructose-1,6bis-phosphate $\cdot$ FAS, fatty acid synthase $\cdot$ FL, $N_{\varepsilon}$-fructosyl- lysine - G-3-P, glycerol-3-phosphate · G-6-P, glucose-6phosphate - GA3P, glyceraldehyde-3-phosphate - GA3PDH, glyceraldehyde-3-phosphate dehydrogenase - GFAT, glutamine:fructose-6-phosphate amidotransferase - GLUT4, insulin-stimulated glucose transporter - GPDH, glycerol-3phosphate dehydrogenase $\cdot$ LPL, lipoprotein lipase $\cdot$ MetSO, methionine sulphoxide $\cdot \mathrm{MG}-\mathrm{H} 1$, methylglyoxal-derived hydroimidazolone $N_{\delta^{-}}$(5-hydro-5-methyl-4-imidazolon-2-yl)ornithine · OGT, UDP- $N$-acetylglucosaminyl transferase $\cdot \mathrm{PH}$, pyruvate dehydrogenase $\cdot$ R-5-P, ribose-5-phosphate $\cdot$ SGLT, sodium-glucose co-transporter $\cdot$ STZ, streptozotocin · TK, transketolase $\cdot$ TMP, thiamine monophosphate $\cdot$ TPI, triosephosphate isomerase $\cdot$ TPP, thiamine pyrophosphate $\cdot$ TPPK, thiamine pyrophosphokinase - TTP, thiamine triphosphate - UDP-GlcNAc, uridine diphosphosphate-Nacetylglucosamine. 


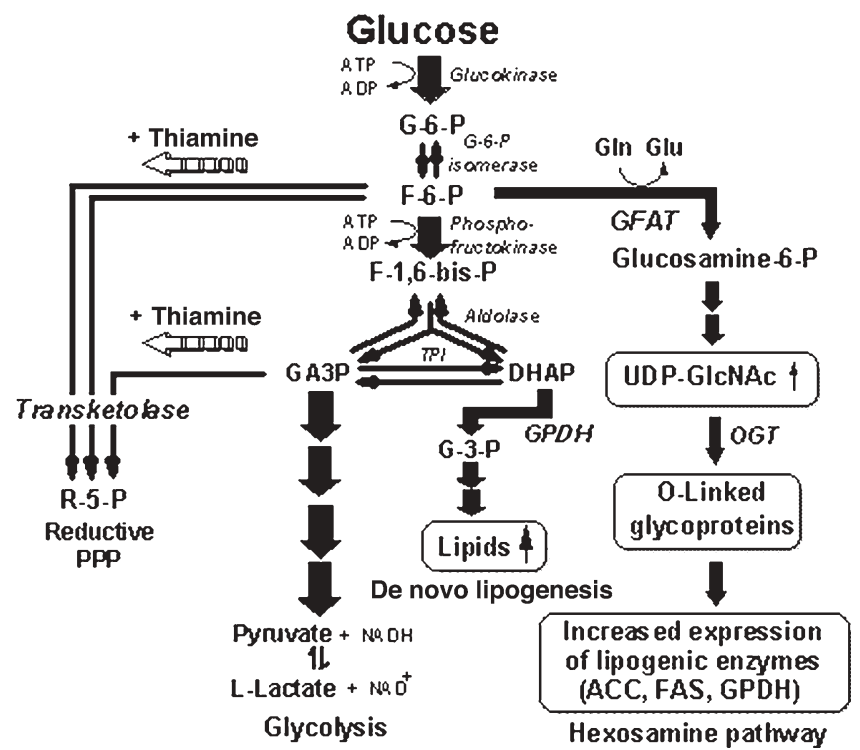

Fig. 1. Metabolic mechanism for the suppression by thiamine of hepatic lipogenesis in diabetes

\section{Introduction}

Diabetes mellitus is associated with a two- to threefold increased risk of CHD in men and a three- to five-fold increase in women, relative to the non-diabetic population. CHD risk determinants in the diabetic population include hyperglycaemia, hyperinsulinaemia in insulin resistance, systolic hypertension, low-grade inflammation, increased triglycerides, cholesterol and plasminogen activator inhibitor-1 $[1,2]$. Dyslipidaemia is a crucial feature in diabetic CHD where increased levels of VLDL-1 particles initiate the development of small dense LDL and HDL particles that pose the main atherogenic threat [3]. Increased lipoprotein secretion by the liver, preceded by a switch from lipid oxidation to lipogenesis and increased lipoprotein synthesis, appears to be central to the development of dyslipidaemia.

The importance of the hexosamine pathway in hyperlipidaemia was indicated recently. In the liver of transgenic mice, overexpression of glutamine:fructose-6-phosphate amidotransferase (GFAT), the ratelimiting enzyme in the hexosamine pathway, was associated with hyperlipidaemia [4]. The glucose-mediated induction of lipogenic enzymes, glycerophosphate dehydrogenase (GPDH), fatty acid synthase (FAS) and acetyl-CoA carboxylase (ACC), was stimulated in liver and adipocytes by activation of the hexosamine pathway [5]. If flux through the hepatic hexosamine pathway is important in lipogenesis in diabetes, high-dose therapy with thiamine and benfotiamine is expected to counter this effect. High-dose thiamine therapy induces the expression of transketolase (TK) and saturates TK with thiamine pyrophosphate (TPP) cofactor, increasing the activity of TK and activating the reductive pentose phosphate pathway. This diverts metabolic flux away from the hexosamine pathway [6] (Fig. 1). To test this hypothesis, we studied the effect of high-dose thiamine and benfotiamine therapy on dyslipidaemia in a rat model of diabetes, namely the streptozotocin (STZ)-induced diabetic rat with maintenance insulin therapy.

We report herein that high-dose thiamine therapy reversed the activation of the hepatic hexosamine pathway and normalised non-HDL cholesterol and triglycerides in STZ diabetic rats. This provides new insight into metabolic signalling to counter dyslipidaemia in diabetes.

\section{Materials and methods}

STZ diabetic rats. A detailed description of the research design was given previously [6]. Briefly, male Sprague-Dawley rats, $250 \mathrm{~g}$, were purchased from Charles River UK (Ramsgate, Kent, UK). They were kept two per cage at $21^{\circ} \mathrm{C}, 50-80 \%$ humidity with a daily 14-h light cycle, and had free access to food and water. Diabetes was induced by injection i.v. with $55 \mathrm{mg} / \mathrm{kg} \mathrm{STZ}$. Body weight and moderate hyperglycaemia were stabilised by s.c. injection of $2 \mathrm{U}$ of Ultralente insulin every 2 days. Thiamine and benfotiamine were given orally, mixed with the chow, at high doses ( 7 and $70 \mathrm{mg} / \mathrm{kg} /$ day) over 24 weeks to STZ diabetic and normal control rats. Body weight and food intake were measured and the chow content of thiamine and benfotiamine for rats receiving the dosing adjusted where necessary to maintain the indicated dose. The diabetic state was characterised by measurement of plasma glucose concentrations and $\mathrm{HbA}_{1}$. Plasma glucose concentrations were determined by the glucose oxidase method and glycated haemoglobin $\mathrm{HbA}_{1}$ by boronate affinity chromatography (diagnostic kits 510 and 442; Sigma, Poole, UK). We assessed ketone bodies in the urine periodically by dip-stick Ketostix testing. No accumulation of ketone bodies was found by this method. After 6,12 and 18 weeks of diabetes, blood samples $(0.2 \mathrm{ml})$ were taken from the tail vein, and at 24 weeks, immediately after the rats were killed, a blood sample was taken by cardiac puncture, with heparin anticoagulant. Blood samples were centrifuged (2000 $\mathrm{g}, 10 \mathrm{~min}$ ) and plasma removed and stored at $-80^{\circ} \mathrm{C}$ until analysis. All procedures were approved by the UK Home Office for work under the Animals (Scientific Procedures) Act 1986; project licence $80 / 1481$. Thiamine hydrochloride and benfotiamine (98-100\%, pharmaceutical grade) were from Sigma and Welding (Hamburg, Germany) respectively.

Plasma cholesterol, HDL cholesterol, triglycerides, UDP$N$-acetylglucosamine and hepatic fatty acid synthase activity. Total plasma cholesterol and triglycerides were determined by endpoint enzymatic assay with INFINITY cholesterol and triglycerides reagents (ThermoTrace, Victoria, Australia). HDL cholesterol was determined by the L-Type HDL-C assay test kit (Wako, Osaka, Japan). Hepatic UDP- $N$-acetylglucosamine (UDP-GlcNAc, nmol/g wet weight) was determined by anion exchange HPLC with spectrophotometric detection [7] and FAS activity was determined spectrophotometrically by measuring the malonyl-CoA- and acetyl-CoA-dependent oxidation of NADPH as described [8].

Transketolase activity, thiamine metabolites, fructosyl-lysine and advanced glycation endproducts and markers of oxidative 


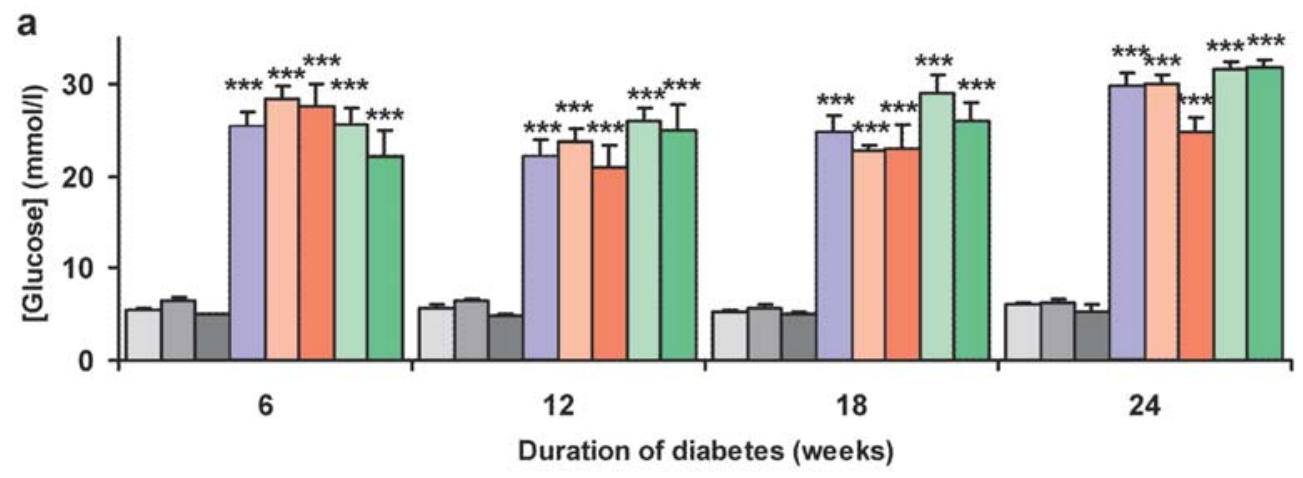

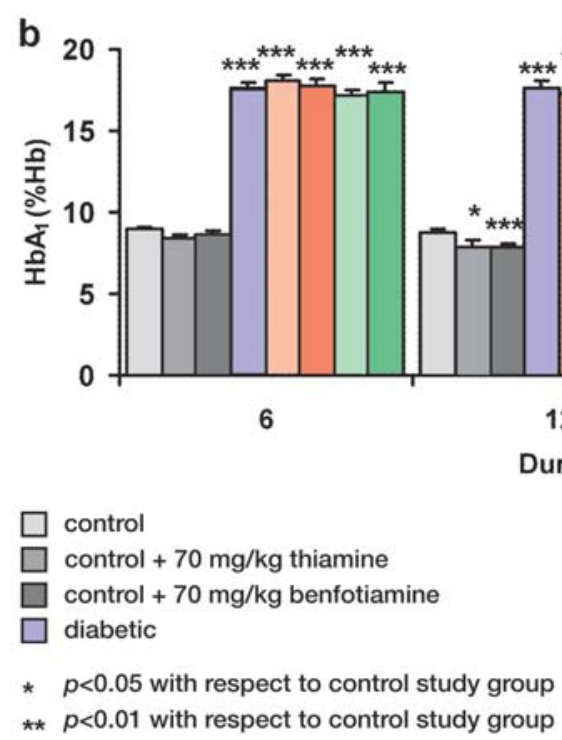

Fig. 2. Effect of high-dose thiamine and benfotiamine therapy on glycaemic control in STZ diabetic rats and controls. a. Blood plasma glucose concentration and b. glycated haemoglobin $\mathrm{HbA}_{1}$. Data are means \pm SEM (n=6-13). $* p<0.05$ with respect to control study group; $* * p<0.01$ with respect to control study group; $* * * p<0.001$ with respect to control study group; ${ }^{0} p<0.05$ with respect to diabetic control study group

stress. Hepatic transketolase (TK) activity was determined by spectrophotometric assay, and concentrations of thiamine, thiamine monophosphate (TMP) and thiamine pyrophosphate (TPP) were determined by HPLC with fluorimetric detection after pre-column derivatisation to thiochromes, as described [6]. Methylglyoxal-derived AGE, the hydroimidazolone MG$\mathrm{H} 1$ and $N_{\varepsilon}$-carboxyethyl-lysine (CEL), the glycoxidation product $\mathrm{N}_{\varepsilon}$-carboxymethyl-lysine (CML), $N_{\varepsilon}$-fructosyl-lysine (FL) and the protein oxidative marker methionine sulphoxide (MetSO) were determined in liver cytosolic protein extracts by enzymatic hydrolysis and liquid chromatography triple quadrupole mass spectrometry with stable isotope-substituted standard calibration, as described $[6,9]$. The concentration of hepatic reduced glutathione $(\mathrm{GSH})$ was determined as nonprotein thiols by derivatisation of deproteinised extracts by Ellman's reagent [10].

Statistical analysis. The significance of differences in mean values of study group analytes was assessed by Student's $t$ test with or without correction for unequal variances (as indicated

\section{Results}

Dyslipidaemia in streptozotocin-induced diabetic rats with maintenance insulin therapy. Effect of high-dose thiamine and benfotiamine therapy. The STZ diabetic rats in this study had frank hyperglycaemia, as indicated by a five-fold increased plasma glucose concentration and two-fold increased glycated haemoglobin $\mathrm{HbA}_{1}$ throughout the study period. Neither thiamine nor benfotiamine therapy improved the glycaemic status of STZ diabetic rats in this study-except for a small decrease in $\mathrm{HbA}_{1}$ with $70 \mathrm{mg} / \mathrm{kg}$ thiamine at week 24 (Fig. 2a, b). STZ diabetic rats were also thiamine deficient. The plasma thiamine concentration was 54\% lower than in normal controls. In STZ diabetic rats plasma thiamine concentration was increased 2 -fold by $7 \mathrm{mg} / \mathrm{kg}$ thiamine. In normal control and STZ diabetic rats it was increased two-fold and 

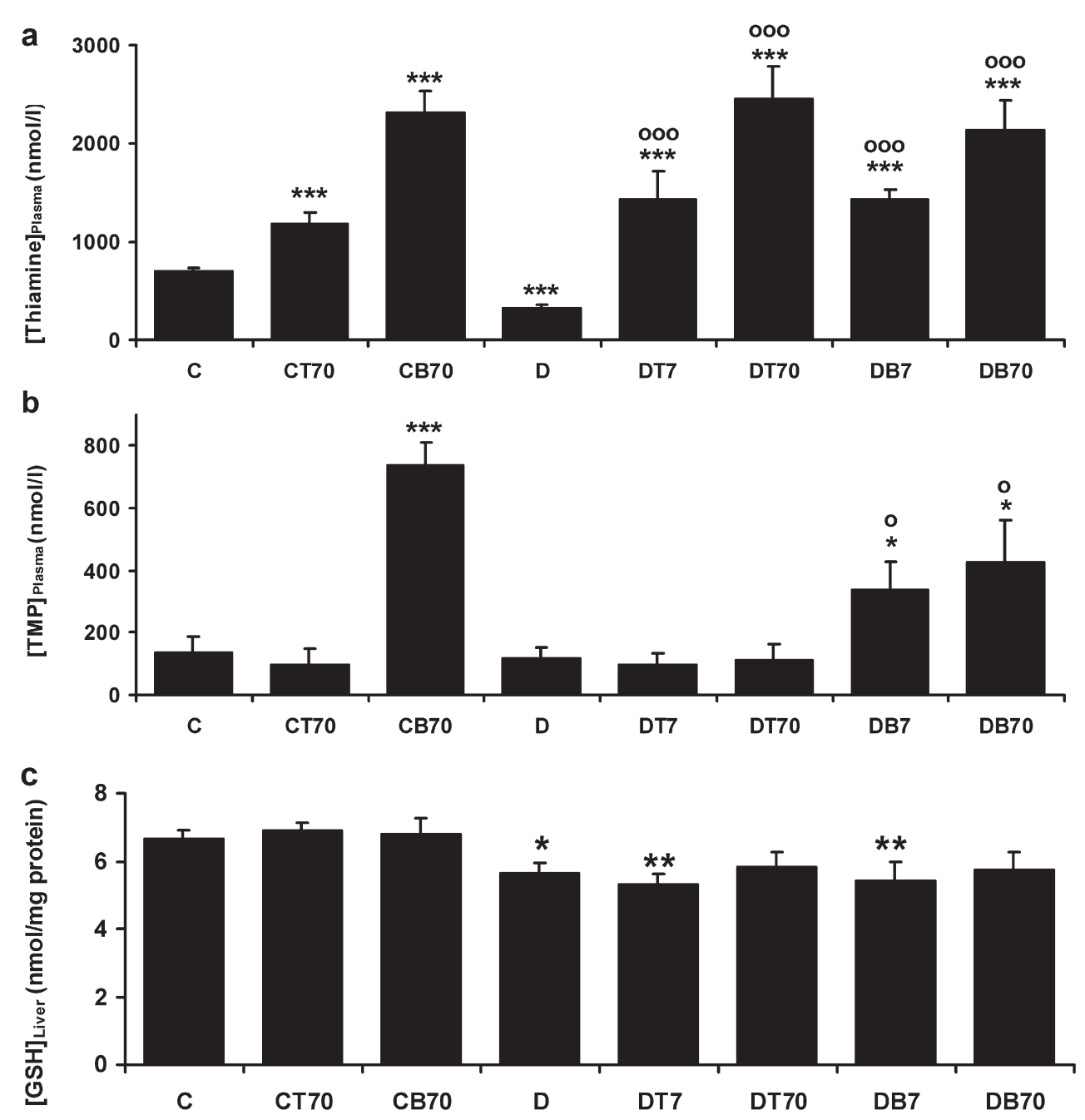

Fig. 3. Effect of high-dose thiamine and benfotiamine therapy on plasma thiamine and hepatic glutathione status in STZ diabetic rats and controls. a. Plasma thiamine concentration, b. plasma thiamine monophosphate concentration, and c. hepatic GSH concentration. C, control; CT70, control $+70 \mathrm{mg} / \mathrm{kg}$ thiamine; CB70, control $+70 \mathrm{mg} / \mathrm{kg}$ benfotiamine; $\mathrm{D}$, diabetic; DT7, diabetic $+7 \mathrm{mg} / \mathrm{kg}$ thiamine; DT70, diabetic $+70 \mathrm{mg} / \mathrm{kg}$ thiamine; DB7, diabetic $+7 \mathrm{mg} / \mathrm{kg}$ benfotiamine; DB70, diabetic $+70 \mathrm{mg} / \mathrm{kg}$ benfotiamine. Data are means \pm SEM $(n=6-13) . * p<0.05$ with respect to control study group; ** $p<0.01$ with respect to control study group; $* * * p<0.001$ with respect to control study group; ${ }^{0} p<0.05$ with respect to diabetic control study group; $000 \quad p<0.001$ with respect to diabetic control study group

three-fold respectively by $70 \mathrm{mg} / \mathrm{kg}$ thiamine, with respect to normal controls $(p<0.001)$. Thiamine therapy did not increase plasma TMP concentrations. Plasma thiamine concentration in STZ diabetic rats was increased two-fold by $7 \mathrm{mg} / \mathrm{kg}$ benfotiamine. It was increased three-fold in normal controls and STZ diabetic rats by $70 \mathrm{mg} / \mathrm{kg}$ benfotiamine, with respect to normal controls $(p<0.001)$. Benfotiamine therapy also increased plasma TMP concentration. Plasma TMP concentration was increased two-fold in STZ diabetic rats by $7 \mathrm{mg} / \mathrm{kg}$ benfotiamine $(p<0.05)$ and five-fold in normal controls $(p<0.001)$ and three-fold in STZ diabetic rats $(p<0.05)$ by $70 \mathrm{mg} / \mathrm{kg}$ benfotiamine, with respect to normal controls $(p<0.001)$ (Fig. 3a, b).

In control rats, the concentration of total plasma cholesterol was $1.95 \pm 0.41 \mathrm{mmol} / \mathrm{l}$, HDL $0.63 \pm 0.11 \mathrm{mmol} / \mathrm{l}$ and triglycerides $1.62 \pm 0.53 \mathrm{mmol} / \mathrm{l}$ (mean $\pm \mathrm{SD}, n=13$ ). In STZ diabetic rats, we found characteristic diabetic dyslipidaemia: the plasma cholesterol was increased $36 \%$, HDL cholesterol decreased $27 \%$ and non-HDL cholesterol increased $69 \%$, with respect to normal controls. Plasma triglycerides were increased $307 \%$, with respect to normal controls (Fig. 4a-d). These characteristics of dyslipidaemia in STZ diabetic rats are similar to those found by other investigators [11] and corroborate well with estimates of plasma cholesterol fractionated by ultracentrifugation techniques [12]. Plasma cholesterol and non-HDL cholesterol were decreased markedly by thiamine therapy $(70 \mathrm{mg} / \mathrm{kg})$. Non-HDL cholesterol was decreased to $29 \%$ below normal control levels. HDL cholesterol was not changed significantly by thiamine therapy. High-dose thiamine $(70 \mathrm{mg} / \mathrm{kg})$ also normalised triglycerides. The TMP derivative, benfotiamine $(70 \mathrm{mg} / \mathrm{kg})$, had no significant effect (Fig. 4a-d). A lower dose of thiamine and benfotiamine 

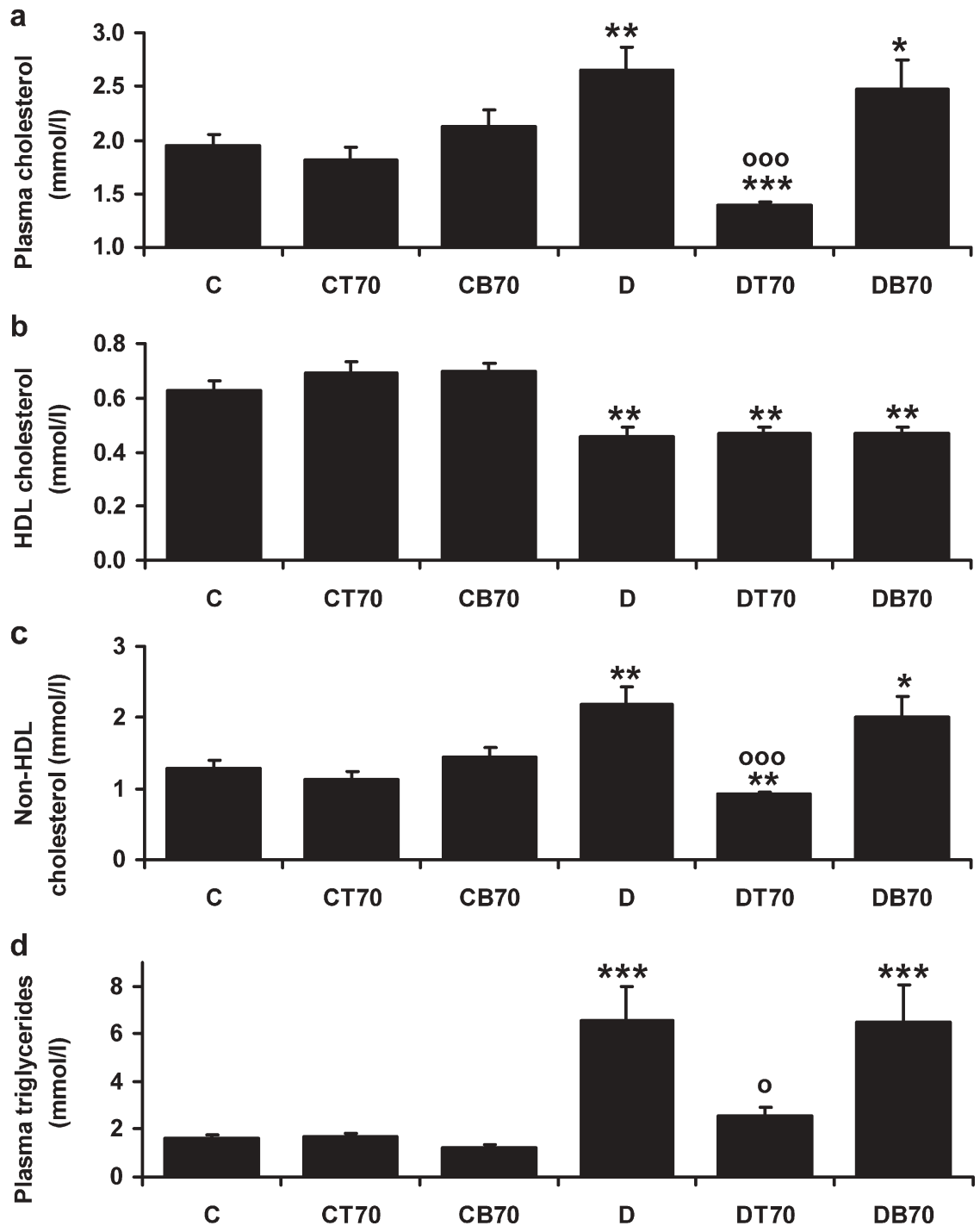

Fig. 4. Reversal of increased plasma cholesterol and triglycerides in streptozotocin-induced diabetic rats by high-dose thiamine therapy. a. Plasma cholesterol, (b) HDL cholesterol, (c) Non-HDL cholesterol and (d) triglycerides. Key: see Fig. 3. Data are means \pm SEM $(n=6-13)$. * $p<0.05$ with respect to control study group; $* * p<0.01$ with respect to control study group; $* * * \quad p<0.001$ with respect to control study group; ${ }_{0} p<0.05$ with respect to diabetic control study group; ${ }^{000} p<0.001$ with respect to diabetic control study group

$(7 \mathrm{mg} / \mathrm{kg}$ ) had no significant effect on plasma cholesterol or triglycerides.

The effect of high-dose thiamine therapy $(70 \mathrm{mg} / \mathrm{kg})$ on the time course of development of diabetic dyslipidaemia was investigated. Throughout the 24 weeks of study, plasma cholesterol and triglyceride concentrations were increased significantly in the STZ diabetic control group, with respect to the normal control group and STZ diabetic group with thiamine therapy $(p<0.001$, Kruskal-Wallis test). At weeks 6, 18 and 24 , plasma cholesterol concentration was increased in the STZ diabetic group but not in the STZ diabetic

group treated with thiamine, with respect to normal controls. At weeks 12, 18 and 24, plasma triglyceride concentration was increased in the STZ diabetic group but not in the STZ diabetic group treated with thiamine, with respect to normal controls (Fig. 5a, b).

The increased absorption of cholesterol esters from the intestinal tract in STZ diabetic rats sustains hypercholesterolaemia of STZ diabetics rats [12] and therefore the food consumption of STZ diabetic rats in this study was of interest. The food consumption of STZ diabetic rats was increased $62 \%$ with respect to normal controls. This was normalised by thiamine therapy ( 7 and $70 \mathrm{mg} / \mathrm{kg}$ ) but not by benfotiamine therapy ( 7 and $70 \mathrm{mg} / \mathrm{kg}$ ). Neither thiamine nor benfotiamine therapy increased the body weight of STZ diabetic rats. Glucosuria of STZ diabetic rats was decreased significantly by high-dose therapy with thiamine $(70 \mathrm{mg} / \mathrm{kg}$ ) and benfotiamine $(7 \mathrm{mg} / \mathrm{kg}$ and $70 \mathrm{mg} / \mathrm{kg}$ ) although plasma glucose concentration was not changed by either agent at both doses. Both thiamine and benfotiamine decreased diuresis in a dose-dependent manner (Table 1). 

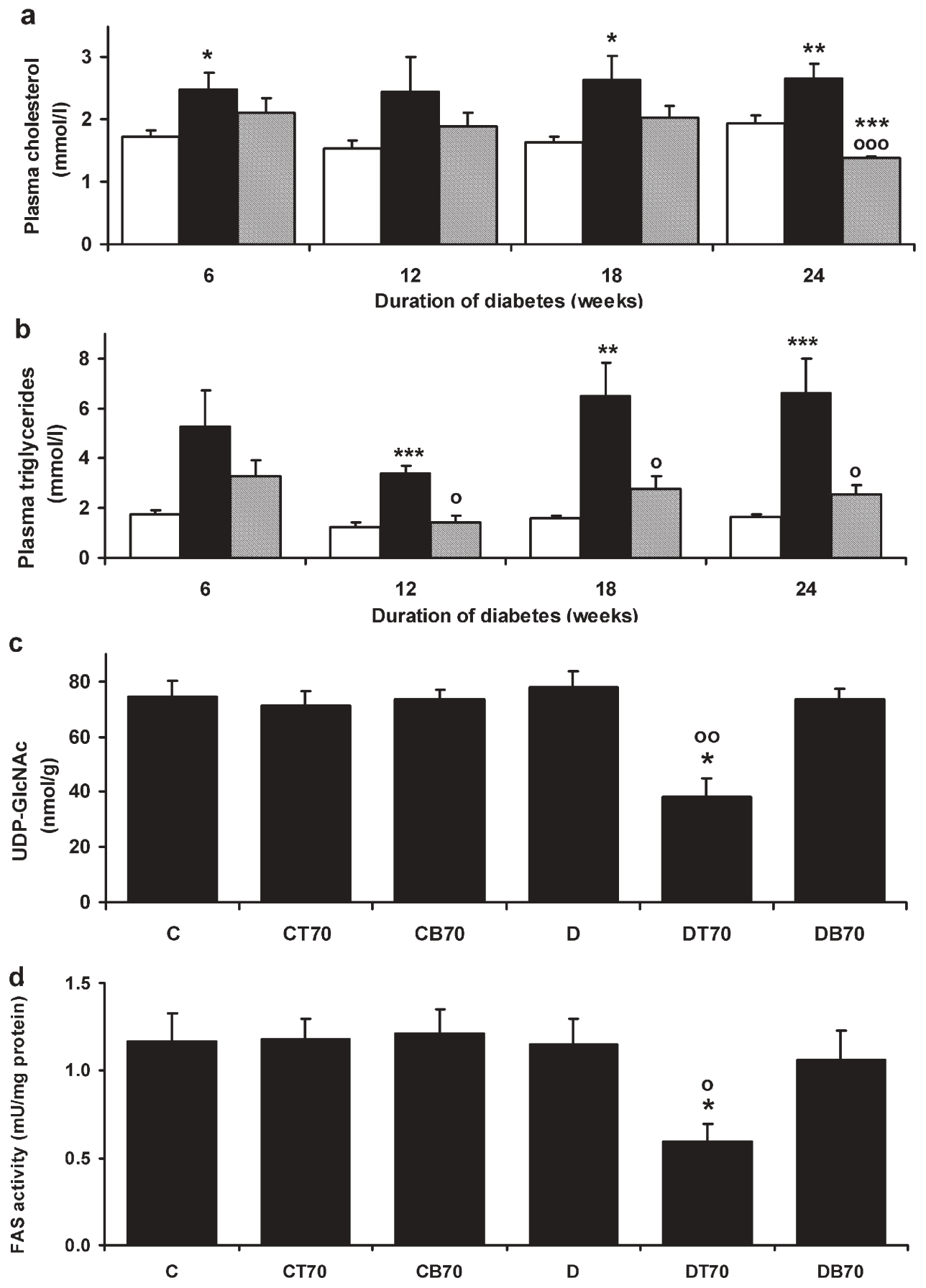

Fig. 5. Time course of changes in plasma cholesterol (a) and triglycerides (b), and hepatic $N$-acetylglucosamine concentration (c) and fatty acid synthase activity (FAS) (d) of streptozotocin-induced diabetic rats with high-dose thiamine therapy. Key $(\mathbf{a}, \mathbf{b})$ : white bar = control; black bar = diabetic; grey bar $=$ diabetic $+70 \mathrm{mg} / \mathrm{kg}$ thiamine. Key $(\mathbf{c}, \mathbf{d})$ : see Figure 3. Data are means \pm SEM $(n=6-13)$. $* p<0.05$ with respect to control study group; $* * \quad p<0.01$ with respect to control study group; $* * * p<0.001$ with respect to control study group; ${ }^{0} p<0.05$ with respect to diabetic control study group; ${ }^{00} p<0.01$ with respect to diabetic control study group; ${ }^{000} p<0.001$ with respect to diabetic control study group

The recent evidence for the involvement of the hexosamine pathway in hepatic lipogenesis led us to investigate the effect of high-dose thiamine on the hepatic concentration of UDP-GlcNAc, a critical intermediate involved in hexosamine pathway signalling [5]. Reversal of diabetic dyslipidaemia by high-dose thiamine was associated with a marked depletion of hepatic UDP-GlcNAc (Fig. 5c) and a 51\% decrease in hepatic FAS activity (Fig. 5d). The hepatic activity of TK was decreased $31 \%$ in STZ diabetic rats, with respect to normal controls, and was normalised by highdose thiamine therapy activity (Fig. 6a). Hepatic TK activity was similar, however, in thiamine- and benfotiamine-treated rats. We investigated the hepatic con- 
Table 1. Body weight, food consumption, glucosuria and diuresis of STZ diabetic and control rats with high-dose thiamine and benfotiamine therapy

\begin{tabular}{|c|c|c|c|c|c|}
\hline $\begin{array}{l}\text { Study } \\
\text { group }\end{array}$ & $n$ & $\begin{array}{l}\text { Body weight at } 24 \\
\text { weeks (g) }\end{array}$ & $\begin{array}{l}\text { Food consumption } \\
\text { (g/day) }\end{array}$ & Glucosuria (mmol/24 h) & Urine volume $(\mathrm{ml} / 24 \mathrm{~h})$ \\
\hline $\mathrm{C}$ & 13 & $664 \pm 88$ & $34 \pm 2$ & $0.021 \pm 0.007$ & $15 \pm 6$ \\
\hline CB70 & 8 & $675 \pm 66$ & $31 \pm 1(p<0.01)$ & $0.008 \pm 0.003(p<0.001)$ & $16 \pm 5$ \\
\hline $\mathrm{D}$ & 11 & $343 \pm 47(p<0.001)$ & $55 \pm 11(p<0.001)$ & $72.8 \pm 23.6(p<0.001)$ & $248 \pm 30(p<0.001)$ \\
\hline DT7 & 7 & $294 \pm 46(p<0.001)$ & $32 \pm 3\left(p^{\prime}<0.001\right)$ & $51.8 \pm 19.6(p<0.001)$ & $170 \pm 75\left(p<0.001 ; p^{\prime}<0.05\right)$ \\
\hline DB70 & 8 & $380 \pm 51(p<0.001)$ & $56 \pm 7(p<0.001)$ & $20.6 \pm 8.7\left(p<0.001 ; p^{\prime}<0.001\right)$ & $75 \pm 29\left(p<0.001 ; p^{\prime}<0.001\right)$ \\
\hline
\end{tabular}

$\mathrm{C}$, control; CT70, control $+70 \mathrm{mg} / \mathrm{kg}$ thiamine; CB70, control $+70 \mathrm{mg} / \mathrm{kg}$ benfotiamine; D, diabetic; DT7 and DT70, diabetic +7 and $70 \mathrm{mg} / \mathrm{kg}$ thiamine; DB7 and DB70, diabetic +7 and $70 \mathrm{mg} / \mathrm{kg}$ benfotiamine DT70. Data are means \pm SD. Signifi-

cance, body weights: $p$ is with respect to 24-week normal controls; significance, food consumption and urine volume: $p$ and $p^{\prime}$ are with respect to normal control and diabetic controls respectively

Table 2. Hepatic protein glycation and oxidation marker residues of streptozotocin-induced diabetic rats and normal controls. Effect of high-dose thiamine therapy

\begin{tabular}{lllll}
\hline Analyte & $\mathrm{C}$ & CT70 & D & DT70 \\
\hline MG-H1 (mmol/mol arg) & $3.34 \pm 0.32$ & $3.21 \pm 0.66$ & $3.35 \pm 0.66$ & $2.47 \pm 0.46^{* *, 0}$ \\
CEL (mmol/mol lys) & $0.14 \pm 0.03$ & $0.15 \pm 0.04$ & $0.13 \pm 0.03$ & $0.06 \pm 0.02^{* * *, 000}$ \\
CML (mmol/mol lys) & $0.10 \pm 0.03$ & $0.13 \pm 0.03$ & $0.17 \pm 0.02^{* *}$ & $0.18 \pm 0.06^{* *}$ \\
FL (mmol/mol lys) & $0.84 \pm 0.43$ & $0.94 \pm 0.19$ & $2.06 \pm 0.55^{* *}$ & $1.92 \pm 0.45^{* *}$ \\
MetSO (mmol/mol met) & $1.92 \pm 0.88$ & $2.70 \pm 0.68$ & $1.85 \pm 0.98$ & $1.89 \pm 1.12$ \\
\hline
\end{tabular}

For key see Table 1. Data are means \pm SD. Significance: * and o denote significance with respect to control and diabetic rats respectively, with one, two and three symbols denoting $p<0.05, p<0.01$ and $p<0.001$ respectively (Mann-Whitney $U$ test)

centrations of thiamine and its metabolites TMP and TPP in diabetic rats and controls. The concentration of TPP was decreased 53\% in STZ diabetic rats, with respect to normal controls (Fig. 6b). This was normalised by both thiamine and benfotiamine therapy. High-dose thiamine therapy increased the concentration of thiamine markedly in the liver of normal and STZ diabetic rats. High-dose benfotiamine therapy was less effective in loading the liver of STZ diabetic rats with thiamine (Fig. 6c). High-dose therapy with benfotiamine rather increased TMP in both control and STZ rats, whereas high-dose thiamine therapy did not. The increase in total hepatic thiamine metabolites (thiamine + TMP + TPP) in STZ diabetic rats was higher in thiamine-treated rats than in benfotiaminetreated rats (Fig. 6b-d).

Increased activity of TK, produced by saturation of TK with TPP substrate and induction of TK expression by high-dose thiamine therapy [6], leads to activation of the reductive pentose phosphate pathway. This may reverse the diabetes-induced increase in flux of triosephosphates, decreasing the rate of formation of the glycating agent methylglyoxal and hence decreasing the levels of related AGE derived from methylglyoxal, MG-H1 and CEL [6]. We determined the concentrations of the methylglyoxal-derived AGE in cytosolic protein extracts of liver homogenates.
Both methylglyoxal-derived AGE were unchanged in cytosolic proteins of diabetic rats, with respect to normal controls, but they were decreased in STZ diabetic rats by high-dose thiamine therapy. In contrast, the glycoxidation product CML and the early glycation adduct FL were increased significantly in diabetic controls and were not changed significantly by thiamine therapy (Table 2). Markers of oxidative stress were also determined. Hepatic GSH concentration was decreased $15 \%$ in STZ diabetic rats, with respect to normal controls. This was prevented by $70 \mathrm{mg} / \mathrm{kg}$ but not $7 \mathrm{mg} / \mathrm{kg}$ thiamine and benfotiamine (Fig. 3c). The concentration of MetSO residues in hepatic protein extracts was not changed significantly in any study group, with respect to normal controls (Table 2).

\section{Discussion}

The effect of high-dose thiamine therapy on diabetic dyslipidaemia has not been investigated previously. The finding that high-dose thiamine therapy reversed and decreased non-HDL cholesterol and normalised triglycerides provides new insight into the physiological regulation of lipoprotein metabolism. This effect was achieved and maintained throughout the study period. The dose of thiamine to achieve these effects, 

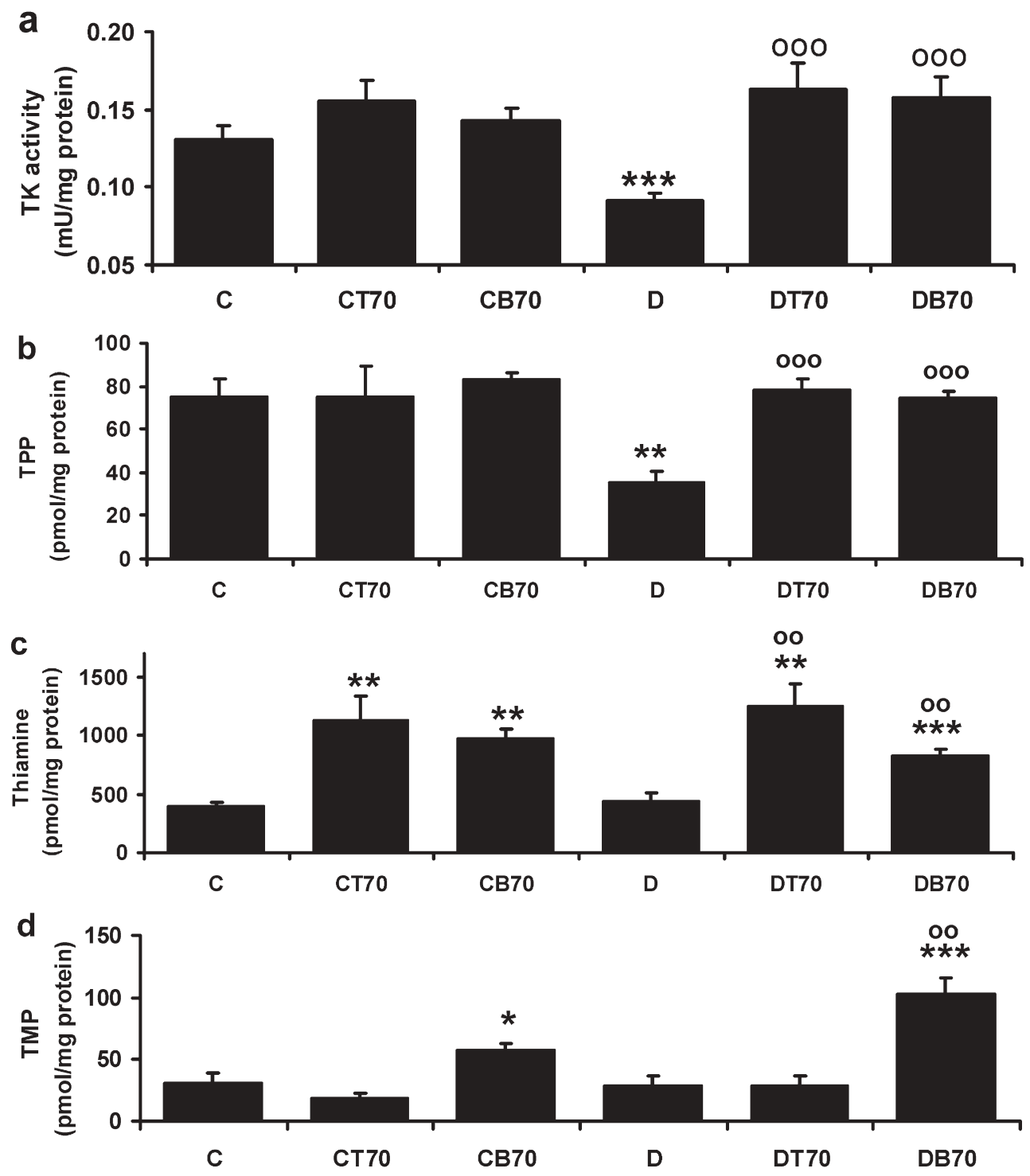

Fig. 6. Hepatic transketolase (TK) activity (a) and thiamine status of streptozotocin-induced diabetic rats with high-dose thiamine therapy. Concentrations of thiamine pyrophosphate (b), thiamine (c) and thiamine monophosphate (d). Key: see Figure 3. Data are means \pm SEM $(n=6-13)$. $* p<0.05$ with respect to control study group; $* * p<0.01$ with respect to control study group; $* * * p<0.001$ with respect to control study group; $00 p<0.01$ with respect to diabetic control study group; ${ }_{000} p<0.001$ with respect to diabetic control study group

however, was very high $(70 \mathrm{mg} / \mathrm{kg})$. Such doses are safe in human subjects over short dosing periods but no long-term evaluations of safety have been performed [13]. High-dose thiamine therapy may be required in diabetes because intestinal absorption of thiamine may be decreased [14] and renal clearance of thiamine increased [6]. Although the intestinal thiamine transporter is saturated by relatively low doses of thiamine, there is slow passive diffusion of thiamine at high concentrations [15].
There was no improvement of glycaemic control in STZ diabetic rats by high-dose thiamine or benfotiamine therapy in this study, as found in independent investigations [16, 17]. Thiamine therapy has been shown to decrease hyperglycaemia in cirrhosis [18], where hyperglycaemia is linked to insulin resistance of muscle and inadequate insulin secretion by beta cells [19], and in thiamine-responsive megaloblastic anaemia (due to mutated high-affinity thiamine transporter), where hyperglycaemia is linked to impaired insulin secretion [20]. Remedial intervention by thiamine in both cases is likely to involve improved beta cell metabolism and insulin secretion. This is not available in the permanent insulin deficiency of the STZ-diabetic rat model and this is probably why thiamine and benfotiamine usually do not improve glycaemic control. It is not known if thiamine or benfotiamine improves glycaemic control in an animal model of type 2 diabetes. Indeed, dyslipidaemia is not usually a major problem in type 1 diabete mellitus, unless very poorly controlled. The reversal of dyslipi- 
daemia in STZ diabetic rats without intermediacy of insulin suggests this effect may be achievable in both type 1 and type 2 diabetes-but this remains to be evaluated.

The increased absorption of cholesterol esters from the intestinal tract sustained the hypercholesterolaemia of STZ diabetics rats. Inhibition of intestinal uptake of cholesterol by inhibition of acyl coenzyme A: cholesterol acyltransferase activity decreased both plasma cholesterol and triglycerides [12]. The normalisation of food consumption by STZ diabetic rats with high-dose thiamine therapy may contribute to the reversal of dyslipidaemia by decreasing exposure to dietary cholesterol. However, both the $7 \mathrm{mg} / \mathrm{kg}$ and $70 \mathrm{mg} / \mathrm{kg}$ doses of thiamine prevented increase food consumption, whereas only the higher dose reversed dyslipidaemia. A combination of decreased food consumption and intervention in lipogenic signalling in the liver may be required to reverse dyslipidaemia. The lack of effect of benfotiamine on dyslipidaemia and food consumption of STZ diabetic rats suggests that there is a significant dichotomy of effects of high-dose thiamine and highdose benfotiamine in the diabetic state.

Both high-dose thiamine and benfotiamine decreased glucosuria and diuresis in STZ diabetic rats. The mechanisms of these effects are not known. Decreased glucosuria may be linked to decreased washout of glucose by the concomitant decreased diuresis and effects on renal glucose transporters. Decreased diuresis may be linked to reversal of diabetes-induced activation of protein kinase $\mathrm{C}$ by highdose thiamine and benfotiamine [6] and consequent reversal of the inhibition of water re-uptake by aquaporins in renal collecting duct cells $[21,22]$. In any event, STZ diabetic rats with high-dose thiamine therapy consumed less food than control STZ diabetic rats whilst maintaining similar body weights. They also consumed less food than STZ diabetic rats with highdose benfotiamine therapy and had a similar reversal of diabetes-induced glucosuria. This suggests a complex mechanism of action of high-dose thiamine therapy that may be linked to more efficient absorption and/or use of nutrients than in STZ diabetic rats with and without high-dose benfotiamine therapy. Decreased food consumption by STZ diabetic rats with high-dose thiamine therapy may be linked to effects of the thiamine metabolites, TPP and thiamine triphosphate (TTP), on dopamine signalling in the brain related to sensory-specific satiety $[23,24]$. Chronic increased dopamine secretion by TPP and TTP may downregulate the feeding incentive response. Oral dosing of thiamine produced higher amounts of protein-associated thiamine metabolites in the brain of mice than dosing with benfotiamine in the critical postprandial period [25]. This may be why thiamine had a satiation effect but benfotiamine did not. Increased expended energy in thiamine- and benfotiamine-treated rats may be due to increased physical activity and/or increased thermogenesis but these were not characterised in this study.

Both high-dose thiamine and benfotiamine decreased urinary glucose excretion in control rats. Since plasma glucose concentration was not changed, this suggests that both compounds increased the renal reuptake of glucose. Glucose re-absorption in the kidney occurs mainly via sodium-glucose co-transporter (SGLT). The insulin-stimulated glucose transporter (GLUT4) is also expressed in the ascending limb of the loop of Henle and is also induced by vasopressin [26, 27, 28]. Thiamine and benfotiamine-induced decrease in glucosuria may be linked to changes in SGLT and vasopressin-stimulated increase in GLUT4 activity.

Both thiamine and benfotiamine also prevented the development of nephropathy in the STZ diabetic rats in this study at both 7 and $70 \mathrm{mg} / \mathrm{kg}$ doses although only thiamine at the $70 \mathrm{mg} / \mathrm{kg}$ dose prevented dyslipidaemia. This suggests that nephropathy develops independently of dyslipidaemia. Indeed, pre-existing hypercholesterolaemia in a mouse model did not exacerbate glomerular lesions or albuminuria in the development of diabetic nephropathy, although it did lead to increased lipid accumulation in the tubular basement membrane [29].

Reversal of dyslipidaemia by high-dose thiamine was associated with a decrease of the hexosamine intermediate UDP-GlcNAc. Glycosylation of the transcription factor Sp1 by UDP-GlcNAc leads to increased transcription of acetyl-CoA carboxylase, the rate-limiting enzyme for fatty acid synthesis [30], and a switch to hepatic lipogenesis [4]. Although, in agreement with Robinson et al. [7], we did not find an increase of hepatic UDP-GlcNAc in diabetic control subjects, the basal hepatic levels of UDP-GlcNAc may be permissive for de novo lipogenesis with concurrent signalling via sterol regulatory element binding protein-1c and carbohydrate response element binding protein [31]. Suppression of UDP-GlcNAc then decreases Sp1 glycosylation and represses lipogenic enzyme expression. Consistent with this, we found a decrease in the hepatic FAS activity of STZ diabetic rats with high-dose thiamine therapy but not in those with high-dose benfotiamine therapy. Hepatic de novo lipogenesis usually makes a minor direct contribution to triglyceride synthesis; triglyceride synthesis from fatty acids delivered to the liver from tissue lipolysis is a more important source of triglycerides. Metabolic signalling for hepatic de novo lipogenesis is, however, a trigger for increased hepatic lipoprotein synthesis and secretion which, together with increased intestinal absorption of cholesterol [12] and decreased LDL cholesterol processing [32], leads to increased circulating atherogenic lipoproteins. The effects on the hepatic hexosamine pathway may explain, in part, why high-dose thiamine therapy counters dyslipidaemia in STZ diabetic rats effectively.

Thiamine therapy did not reverse decreased plasma HDL concentration in STZ diabetic rats. Increased ex- 
pression of cholesteryl ester transfer protein (CTEP) is linked to decreased HDL levels [33]. This was reversed by intensive insulin therapy [34] but appears not to be under the control of thiamine-induced responses. Decreased lipoprotein lipase (LPL) activity is associated with impaired removal and increased plasma concentrations of triglycerides in diabetes [35]. LPL gene expression is activated through the transcription factor $\mathrm{Sp} 1$ in its unglycosylated state [36]. The decreased glycosylation of Sp1 implicated in repression of lipogenic enzymes is expected to produce a concomitant Sp1-activated increase in LPL expression and activity. Indeed, this may also be involved in the correction of dyslipidaemia by thiamine.

Dysfunctional signalling for hepatic lipogenesis was sustained by decreased TK activity due to depletion of the cofactor TPP, although liver thiamine and TMP levels were normal. Overall, STZ diabetic rats were thiamine deficient because of increased renal clearance of thiamine [6]. This produced a selective decrease in TPP concentration without decreased concentrations of thiamine and TMP. Hepatic thiamine deficiency was reported previously in STZ diabetic Wistar rats [37] and alloxan-induced diabetic mice [38]. In the latter study, thiamine metabolites were analysed and a selective decline in TPP concentration found in the liver and heart. Thiamine enters hepatocytes mainly by the high-affinity thiamine transporter $[39,40]$ and TMP enters cells by the reduced folate transporter [41]. In the cytosol, TMP is hydrolysed rapidly to thiamine, and thiamine is phosphorylated to TPP by thiamine pyrophosphokinase (TPPK) [41]. The expression of TPPK was decreased in the liver and heart of thiamine-deficient rats [42]. Hence, diabetes-induced thiamine deficiency, with associated decreased expression of TPPK, may account for the decrease in hepatic TPP concentration.

Hepatic expression of thiamine-dependent enzymes is sensitive to decreased TPP concentration and leads to decreased TK and pyruvate dehydrogenase $(\mathrm{PH})$ activities [43]. Normalisation of both TK and PH activities may counter hepatic lipogenesis and dyslipidaemia herein. Decreased TK activity impedes the reductive pentose phosphate pathway and thereby permits flux of fructose-6-phosphate to enter the hexosamine pathway and also sustains the metabolic flux for triosephosphate formation (Fig. 1). A small proportion (0.1-1\%) of triosephosphates degrade non-enzymatically to form methylglyoxa, a potent glycating agent and precursor of the AGE, MG-H1 and CEL [9]. High-dose thiamine therapy decreased MG-H1 and CEL residue concentration of cytosolic protein extracts of the liver but not the concentrations of CML or FL residues. This is consistent with the activation of the reductive pentose phosphate pathway and decrease of both fructose-6-phosphate and glyceraldehyde-3-phosphate (and indirectly dihydroxyacetonephosphate) by high-dose thiamine therapy. Markers of oxidative stress indicated only mild oxidative stress in the liver in STZ diabetic rats, which was reversed by thiamine and benfotiamine at the $70 \mathrm{mg} / \mathrm{kg}$ doses.

We cannot exclude a role for methylglyoxal-mediated effects in diabetic dyslipidaemia. Indeed, in independent studies we observed that intraperitoneal injection of methylglyoxal $(50 \mathrm{mg} / \mathrm{kg})$ in non-diabetic rats induced dyslipidaemia characterised by a $63 \%$ increase in plasma cholesterol and an $87 \%$ increase in plasma triglycerides (unpublished observations, J. Berlanga, P.J. Thornalley). Glycation of Sp1 may target the transcription factor for proteasomal degradation and lift the repression of lipogenic enzyme synthesis. Alternatively, glycation of apolipoprotein B100 may confer resistance to proteasomal degradation [44]. Further studies on the effects of glycation on lipogenic signalling and lipoprotein metabolism are required.

It was surprising that the TMP derivative, benfotiamine, did not have similar effects on diabetic dyslipidaemia to those of thiamine since it is thought to be an improved delivery vehicle for thiamine [45]. The reported biodistribution data indicated, however, that exogenous thiamine increased hepatic thiamine levels more markedly than benfotiamine in the 90-minute postfeeding period [25], probably because of efficient uptake of portal venous thiamine into the liver. Postprandial lipidaemia may be particularly important in triggering changes in lipid metabolism in STZ diabetic rats [12]. High-dose thiamine and benfotiamine both normalised liver TPP concentration and hepatic TK activity in diabetic rats. Thiamine dosing may increase hepatic TPP concentration faster than benfotiamine dosing in the postprandial period. Since TK has a short half-life when unsaturated with TPP (25 min) [46], a rapid increase in hepatic TPP concentration may be followed by a prompt increase in TK expression and activity in the postprandial period when dietary cholesterol and triglycerides are absorbed. Hence, lipogenic responses may be repressed by dosing with thiamine when they would otherwise be activated maximally.

The plasma concentration of thiamine in STZ diabetic rats in this study was decreased $54 \%$, with respect to normal controls. This was due to an eight-fold increase in the renal clearance of thiamine in STZ diabetic rats [6]. High-dose thiamine and benfotiamine therapy gave a dose-dependent increase in the plasma concentration of thiamine, both compounds supra-normalising plasma thiamine concentration at both doses in STZ diabetic rats. Benfotiamine is a vehicle for the delivery of TMP and increased plasma TMP concentration in both control and STZ diabetic rats (Fig. 3a, b). High-dose thiamine $(70 \mathrm{mg} / \mathrm{kg})$ increased liver thiamine levels more than benfotiamine $(70 \mathrm{mg} / \mathrm{kg})$. The liver TMP concentration was increased markedly (four-fold) by benfotiamine up to ca. $30 \mathrm{nmol} / \mathrm{g}$ wet weight or more than $75 \mu \mathrm{mol} / \mathrm{l}$ in the hepatic cytosol. High concentrations of TMP inhibit TPPK activity 
non-competitively $\left(\mathrm{K}_{\mathrm{i}}=17 \mu \mathrm{mol} / \mathrm{l}\right)$ [47] and inhibit the entry of TPP into mitochondria competitively $\left(\mathrm{K}_{\mathrm{i}}=150 \mu \mathrm{mol} / \mathrm{l}\right)$ [48]. This may decrease the availability of TPP in diabetic rats. This may limit the effectiveness of benfotiamine and other vehicles of TMP delivery in countering diabetic dyslipidaemia.

High-dose thiamine therapy (7 and $70 \mathrm{mg} / \mathrm{kg}$ ) has recently been shown to prevent incipient diabetic nephropathy [6], where benfotiamine was equally effective, and $70 \mathrm{mg} / \mathrm{kg}$ thiamine also prevented diabetic dyslipidaemia. Thiamine and benfotiamine also suppressed the development of diabetic neuropathy and benfotiamine suppressed the development of retinopathy (where thiamine was not evaluated) in experimental diabetes in vivo [16, 17]. It is not known if high-dose therapy of thiamine or benfotiamine will be effective against microvascular complications, dyslipidaemia and cardiovascular disease in clinical diabetes. Clinical studies to date suggest that a significant proportion of diabetic patients are thiamine deficient [49, 50]. Given the continuing toll of CHD in the diabetic population, and the emerging multiple benefits of thiamine repletion in diabetes, we suggest that even mild thiamine deficiency in diabetes should be avoided and thiamine supplementation to high dose should be considered as a part of nutritional therapy to counter diabetic dyslipidaemia and vascular complications.

Acknowledgements. We thank the Juvenile Diabetes Research Fund (USA) and the Wellcome Trust (UK) for supporting this research.

\section{References}

1. American Diabetes Association (2004) Dyslipidemia management in adults with diabetes. Diabetes Care 27:S71

2. Grant PJ (2003) The genetics of atherothrombotic disorders: a clinician's view. J Thromb Haemost 1:1381-1390

3. Taskinen M-R (2004) Diabetic dyslipidaemia: from basic research to clinical practice. Diabetologia 46:733-749

4. Veerababu G, Tang J, Hoffman RT et al. (2000) Overexpression of glutamine:fructose-6-phosphate amidotransferase in the liver of transgenic mice results in enhanced glycogen storage, hyperlipidemia, obesity, and impaired glucose tolerance. Diabetes 49:2070-2078

5. Rumberger JM, Wu T, Hering MA, Marshall S (2003) Role of hexosamine biosynthesis in glucose-mediated up-regulation of lipogenic enzyme mRNA levels. J Biol Chem 278:28547-28552

6. Babaei-Jadidi R, Karachalias N, Ahmed N, Battah S, Thornalley PJ (2003) Prevention of incipient diabetic nephropathy by high-dose thiamine and Benfotiamine. Diabetes 52:2110-2120

7. Robinson KA, Weinstein ML, Lindenmayer GE, Buse MG (1995) Effects of diabetes and hyperglycemia on the hexosamine synthesis pathway in rat muscle and liver. Diabetes 44:1438-1446

8. Moibi JA, Ekpe ED, Christopherson RJ (2000) Acetyl-CoA carboxylase and fatty acid synthase activity and immunodetectable protein in adipose tissues of ruminants: effect of temperature and feeding level. J Anim Sci 78:2383-2392
9. Thornalley PJ, Battah S, Ahmed N et al. (2003) Quantitative screening of advanced glycation endproducts in cellular and extracellular proteins by tandem mass spectrometry. Biochem J 375:581-592

10. Beutler E (1975) Reduced glutathione. In: Red blood cell metabolism. A manual of biochemical methods, 2nd edn. Grune and Stratton, New York, pp 112-114

11. Degenhardt TP, Alderson NL, Arrington DD et al. (2002) Pyridoxamine inhibits early renal disease and dyslipidemia in the streptozotocin-diabetic rat. Kidney Int 61:939-950

12. Kusunoki J, Aragane K, Kitamine T et al. (2000) Postprandial hyperlipidemia in streptozotocin-induced diabetic rats is due to abnormal increase in intestinal acyl Coenzyme A:cholesterol acyltransferase activity. Arterioscler Thromb Vasc Biol 20:171-178

13. Expert Group on Vitamins and Minerals, Food Standards Agency UK (2003) Safe upper levels for vitamins and minerals, pp 74-80

14. Patrini C, Laforenza U, Gastaldi G, Verri A, Ferrari G, Rindi G (1996) Effects of insulin on thiamine intestinal transport in rat everted jejunal sacs. J Physiol (Lond) 493:100S-101S

15. Rindi G, Laforenza U (2000) Thiamine intestinal transport and related issues: recent aspects. Proc Soc Exp Biol Med 224:246-255

16. Hammes H-P, Du X, Edelstein D et al. (2003) Benfotiamine blocks three major pathways of hyperglycemic damage and prevents experimental diabetic retinopathy. Nat Med 9:294-299

17. Stracke H, Hammes HP, Werkmann D et al. (2001) Efficacy of benfotiamine versus thiamine on function and glycation products of peripheral nerves in diabetic rats. Exp Clin Endocrinol Diabetes 109:330-336

18. Hassan R, Qureshi H, Zuberi SJ (1991) Effect of thiamine on glucose-utilisation in hepatic cirrhosis. J Gastroenterol Hepatol 6:59-60

19. Petrides AS, Vogt C, Schulzeberge D, Matthews D, Strohmeyer G (1994) Pathogenesis of glucose-intolerance and diabetes-mellitus in cirrhosis. Hepatology 19:616-627

20. Oishi K, Hofmann S, Diaz GA et al. (2002) Targeted disruption of Slc19a2, the gene encoding the high-affinity thiamin transporter Thtr-1, causes diabetes mellitus, sensorineural deafness and megaloblastosis in mice. Hum Mol Genet 11:2951-2960

21. Van Balkom BWM, Savelkoul PJM, Markovich D et al. (2002) The role of putative phosphorylation sites in the targeting and shuttling of the aquaporin-2 water channel. J Biol Chem 277:41473-41479

22. Zelenina M, Zelenin S, Bondar AA, Brismar H, Aperia A (2002) Water permeability of aquaporin-4 is decreased by protein kinase $\mathrm{C}$ and dopamine. Am J Physiol Renal Physiol 283:F309-F318

23. Ahn S, Phillips AG (2002) Modulation by central and basolateral amygdalar nuclei of dopaminergic correlates of feeding to satiety in the rat nucleus accumbens and medial prefrontal cortex. J Neurosci 22:10958-10965

24. Yamashita H, Zhang YX, Nakamura S (1993) The effects of thiamin and its phosphate-esters on dopamine release in the rat striatum. Neurosci Lett 158:229-231

25. Hilbig R, Rahmann H (1998) Comparative autoradiographic investigations on the tissue distribution of benfotiamine versus thiamine in mice. Arzneimittelforschung 48:461-468

26. Heilig CW, Zaloga C, Lee $\mathrm{M}$ et al. (1995) Immunogold localization of high-affinity glucose transporter isoforms in normal rat kidney. Lab Invest 73:674-684

27. Chin E, Zamah AMZ, Landau D et al. (1997) Changes in facilitative glucose transporter messenger ribonucleic acid 
levels in the diabetic rat kidney. Endocrinology 138: 1267-1275

28. Chin E, Zhou J, Bondy C (1993) Anatomical and developmental patterns of facilitative glucose transporter gene-expression in the rat kidney. J Clin Invest 91:1810-1815

29. Hammad SM, Hazen-Martin DJ, Sohn M et al. (2003) Nephropathy in a hypercholesterolemic mouse model with streptozotocin-induced diabetes. Kidney Blood Press Res 26:351-361

30. Daniel S, Kim K-H (1996) Sp1 mediates glucose activation of the acetyl-CoA carboxylase promoter. J Biol Chem 271:1385-1392

31. Foufell F, Ferre P (2002) New perspectives in the regulation of hepatic glycolytic and lipogenic genes by insulin and glucose: a role for the transcription factor sterol regulatory element binding protein-1c. Biochem J 366:377-391

32. McLean MP, Billheimer JT, Warden KJ, Irby RB (1995) Differential expression of hepatic sterol carrier proteins in the streptozotocin-treated diabetic rat. Endocrinology 136:3360-3368

33. Cheema SK, Rashid-Kolvear F (2003) Streptozotocin-induced increase in cholesterol ester transfer protein (CETP) and its reversal by insulin in transgenic mice expressing human CETP. Can J Physiol Pharmacol 81:997-1004

34. Berti JA, Casquero AC, Patricio PR et al. (2003) Cholesteryl ester transfer protein expression is down-regulated in hyperinsulinemic transgenic mice. J Lipid Res 44:1870-1876

35. Nikkila EA, Huttunen JK, Ehnholm C (1977) Postheparin plasma lipoprotein lipase and hepatic lipase in diabetes mellitus. Relationship to plasma triglyceride metabolism. Diabetes 26:11-21

36. Yang WS, Deeb SS (1998) Sp1 and Sp3 transactivate the human lipoprotein lipase gene promoter through binding to a CT element: synergy with the sterol regulatory element binding protein and reduced transactivation of a naturally occurring promoter variant. J Lipid Res 39:2054-2064

37. Reddi AS, Jyothirmayi GN, Deangelis B, Frank O, Baker H (1993) Tissue concentrations of water-soluble vitamins in normal and diabetic rats. Int J Vitam Nutrit Res 63:140-144

38. Hobara R, Kato H, Sakamoto K (1983) Effect of thiamine and thiamine levels on experimental alloxan induced diabetes mellitus. Jpn J Pharmacol 33:27-31
39. Dutta B, Huang W, Molero M et al. (1999) Cloning of the human thiamine transporter, a member of the folate transporter family. J Biol Chem 274:31925-31929

40. Yoshioka K (1984) Some properties of the thiamine uptake system in isolated rat hepatocytes. Biochim Biophys Acta 778:201-209

41. Zhao R, Gao F, Goldman ID (2002) Reduced folate carrier transports thiamine monophosphate: an alternative route for thiamine delivery into mammalian cells. Am J Physiol Cell Physiol 282:C1512-C1517

42. Sanioto SM, Reinauer H, Hollmann S (1977) Thiamine pyrophosphokinase activity in liver, heart and brain crude extracts of control and thiamine deficient rats. Int J Vitam Nutr Res 47:315-324

43. Terbukhina RV, Ostrovsky YM, Petushok VG, Velichko MG, Tumanov VN (1981) Effect of thiamine deprivation on thiamine metabolism in mice. J Nutr 111:505-513

44. Fisher EA, Zhou MY, Mitchell DM et al. (1997) The degradation of apolipoprotein B100 is mediated by the ubiquitinproteasome pathway and involves heat shock protein 70 . J Biol Chem 272:20427-20434

45. Schreeb KH, Freudenthaler S, Vormfelde SV, GundertRemy U, Gleiter CH (1997) Comparative bioavailability of two vitamin B1 preparations: Benfotiamine and thiamine mononitrate. Eur J Clin Pharmacol 52:319-320

46. Soh Y, Song BJ, Jeng JJ, Kallarakal AT (1998) Critical role of $\operatorname{Arg}(433)$ in rat transketolase activity as probed by sitedirected mutagenesis. Biochem J 333:367-372

47. Wakabayashi Y (1978) Purification and properties of porcine thiamine pyrophosphokinase. Vitamins 52: 223-236

48. Barile M, Valenti D, Brizio C, Quagliariello E, Passarella S (1998) Rat liver mitochondria can hydrolyse thiamine pyrophosphate to thiamine monophosphate which can cross the mitochondrial membrane in a carrier-mediated process. FEBS Lett 435:6-10

49. Saito N, Kimura M, Kuchiba A, Itokawa Y (1987) Blood thiamine levels in outpatients with diabetes mellitus. J Nutr Sci Vitaminol 33:421-430

50. Havivi E, Bar On H, Reshef A, Raz I (1991) Vitamins and trace metals status in non insulin dependent diabetes mellitus. Int J Vit Nutr Res 61:328-333 